\title{
Variabilité interspécifique de trois algues rouges : Hypnea musciformis, Gracilaria multipartita et Gelidium sesquipedale (Rhodophycées) de la côte atlantique marocaine
}

\section{Aziza Mouradi', Meryem Chikhaovi', Mohammed Fekhaovi', Rachida Akallal', Amina Guessous' et Thierry Givernaud $^{2}$ \\ 'Laboratoire de biochimie, biotechnologies et environnement, Université Ibn Tofaïl, Faculté des sciences 14000, Kénitra, Maroc \\ ${ }^{2}$ Laboratoire d'hydrobiologie, Département de zoologie, Institut scientifique, Université Mohamed V, Agdal, Rabat, Maroc}

(Reçu le 26 Février 2006, accepté le 11 Juillet 2006)

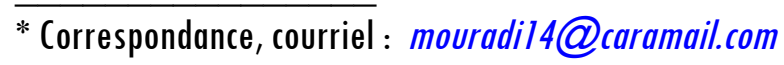

\section{Résumé}

L'analyse globale de la croissance de trois espèces d'algues rouges; Hypnea musciformis, Gracilaria multipartita et Gelidium sesquipedale a été entreprise dans le but de déterminer la variabilité interspécifique au sein du même milieu naturel. Le site étudié est la plage de Mehdia située au nord de la côte atlantique marocaine.

Les résultats montrent des différences spécifiques dans les rythmes de croissance des trois espèces étudiées. Gracilaria multipartita présente un développement important des paramètres de croissance à savoir la longueur, le poids et le nombre total de ramifications, surtout pendant la période estivale par rapport à Gelidium sesquipedale et Hypnea musciformis. Les périodes actives de croissance de Gracilaria multipartita sont caractérisées par des températures et des intensités lumineuses légèrement importantes et par des teneurs élevées en nitrates et en phosphates. La croissance d'Hypnea musciformis montre des fluctuations saisonnières dues aux variations des facteurs environnementaux tels que la lumière, la température et la salinité principalement. Par contre, la croissance de Gelidium sesquipedale est lente et semble être plus liée à des facteurs intrinsèques qu'aux facteurs externes.

Mots-clés : rhodophycées, croissance, variabilité interspécifique, $A C P$, côte atlantique marocaine 


\section{Abstract \\ Interspecific variability of the red algae: Hypnea musciformis, Gracilaria multipartita and Gelidium sesquipedale (Rhodophyceae) of the Moroccan atlantic coast}

Total analysis of the growth of three red algae species; Hypnea musciformis, Gracilaria multipartita and Gelidium sesquipedale were undertaken with an aim of determining interspecific variability within the same natural environment. The studied site is the beach of Mehdia located at the north of the Moroccan Atlantic coast.

The results show specific differences in the growth rates of the three studied species. Gracilaria multipartita presents a significant development of the growth parameters like the size, the weight and the total ramification, especially for the aestival period compared to Gelidium sesquipedale and Hypnea musciformis.

The active growth periods of Gracilaria multipartita are characterized by slightly important temperatures and light intensities and by high percentages of nitrates and phosphates. On the other hand, the growth of Hypnea musciformis shows fluctuations due to the variations of the environmental factors. The growth of Gelidium sesquipedale is slow and seems more under the influence of intrinsic factors than environmental factors.

Keywords : rhodophyceae, growth, interspecific variability, $A C P$, Moroccan atlantic coast.

\section{Introduction}

Les algues marines constituent une source importante de produits utilisés par l'Homme pour son alimentation et pour diverses applications industrielles. Elles contribuent ainsi au développement des activités socio-économiques mondiales; ainsi l'industrie des algues a augmenté de $26 \%$ entre 1993 et 2002 à 6 billions US\$ [1,2]. L'un des groupes les plus importants est celui des algues rouges dont la phase matricielle de la paroi de plusieurs espèces est constituée de polysaccharides de type galactanes plus ou moins sulfatés [3]. Ces polysaccharides forment une famille très complexe dont les représentants les mieux connus sont les agars et les carraghénanes. Ils trouvent de nombreuses applications comme agents texturant, filmogènes ou émulsifiants dans de nombreux domaines de l'industrie agro-alimentaire [4-7] ou pharmaceutique [8-10].

Les principaux macrophytes exploités pour la production d'agar appartiennent à l'ordre des Gélidiales et des Gracilariales [11], ceux utilisés pour leurs extraits de carraghénanes sont principalement des Gigartinales [12]. Le développement actuel de nouvelles applications biotechnologiques et agro-alimentaires sont à l'origine de 
l'augmentation de la demande mondiale en agar et en carraghénanes. II est donc nécessaire de bien gérer les ressources disponibles et de développer l'aquaculture.

De nombreuses études ont montré que la croissance et le développement des algues sont liés principalement à des facteurs physico-chimiques tels que, la lumière, la température, le pH et l'enrichissement du milieu en sels nutritifs et en carbonates [13-17]. En effet, la croissance de la plupart des espèces algales montre des fluctuations saisonnières dues aux variations de ces facteurs environnementaux [18-20].

L'industrie de l'agar a développé, tout au long des côtes du Maroc, une activité qui assure un emploi permanent ou temporaire à plus de 8000 personnes et ramène plus de 20 millions de dollars par an. Cette industrie place ainsi le Maroc parmi les premiers producteurs mondiaux. Cependant, il a été montré que les champs d'agarophytes souffrent d'une surexploitation importante [21] par manque d'une gestion adéquate de ces champs et le non-respect de la législation mise en place dernièrement et qui est basée sur des résultats scientifiques. Des études de repeuplement des champs d'agarophytes [22] ont été réalisées au Maroc et ont montré qu'il est nécessaire, avant l'exploitation d'une espèce, de bien maîtriser la biologique de cette dernière.

A côté des algues agarophytes, coexistent des peuplements importants d'autres espèces d'algues rouges productrices de carraghénanes. Le Maroc récolte et exporte sous forme de matière première 400 à 600 tonnes d'algues carraghénophytes, qui pourraient être traitées localement car l'extraction de ces phycocolloïdes est comparable à celle de l'agar.

Afin de déterminer la meilleure période de récolte et de mieux gérer les champs d'algues exploitables sur les côtes marocaines, le présent travail vise à suivre la croissance et le développement des populations.

Les trois espèces d'Hypnea musciformis (Wulfen) Lamouroux, de Gracilaria multipartita (Clemente) Harvey et de Gelidium sesquipedale (Turner) Thuret ont fait l'objet d'études séparées (El Bacha et al. en 2004 [19], Mouradi et al. (soumis dans la revue "I Afrique Science II) ), afin de déterminer leur cycle de croissance et la meilleure période de récolte pour gérer au mieux leur exploitation. Dans cette publication, on tente de comparer les stratégies de croissances de ces trois espèces, après un rappel sur leurs caractéristiques propres.

\section{Matériel et méthodes}

\section{2-1. Présentation des stations de récolte}

La récolte des trois espèces d'algues rouges, Hypnea musciformis, Gelidium sesquipedale et Gracilaria multipartita, a été effectuée mensuellement à la plage de Mehdia située à $35 \mathrm{~km}$ de la ville de Rabat sur la rive gauche de l'embouchure de l'oved 
Sebou et devant la plaine du Gharb (Figure 1). Le suivi de la croissance en milieu naturel de Gracilaria multipartita et de Gelidium sesquipedale a été effectué au cours de l'année 1996, celui d'Hypnea musciformis a été réalisé en 1997.

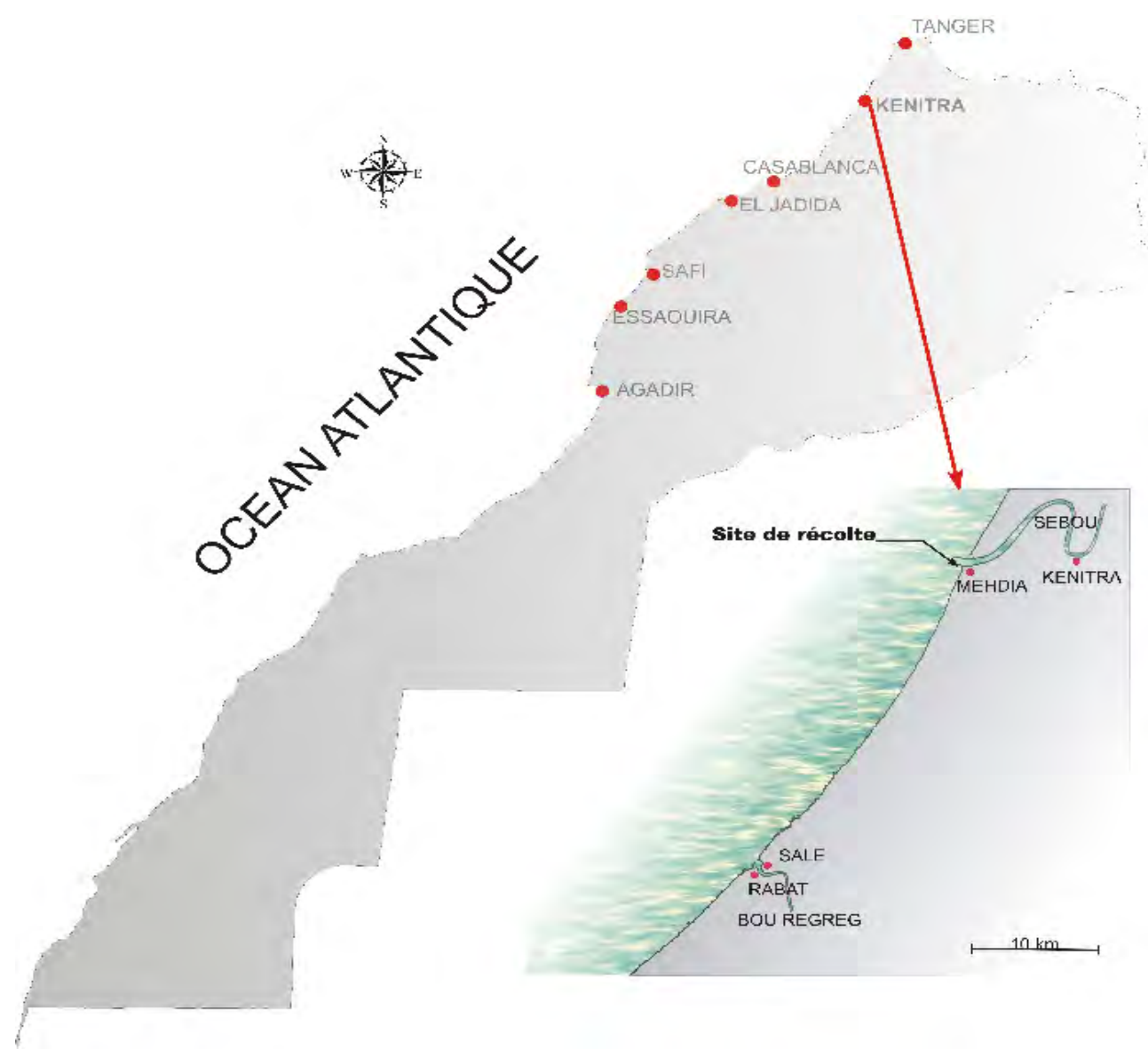

Figure 1 : Situation géographique de la station de récolte.

\section{2-2. Présentation du matériel d'étude}

L'agarophyte Gracilaria multipartita présente une fronde aplatie ramifiée subdicotomiquement (Figure 2). L'identification des gracilaires est très confuse. L'identification de $G$. multipartita a été confirmée par l'étude moléculaire en utilisant la séquence d'ADN codant pour l'ARNr de la petite sous unité du ribosome (18SrDNA) et par le spacer de la rubisco selon la méthode de Goff et Coleman [23]. 

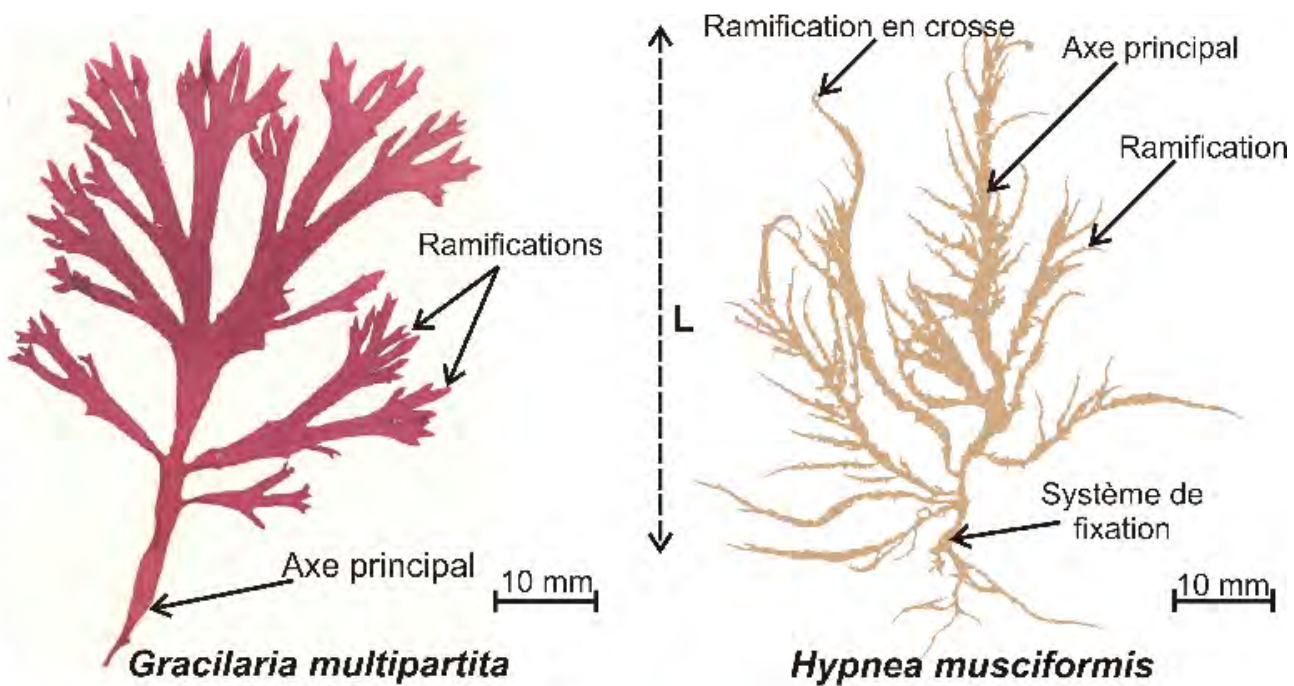

Hypnea musciformis

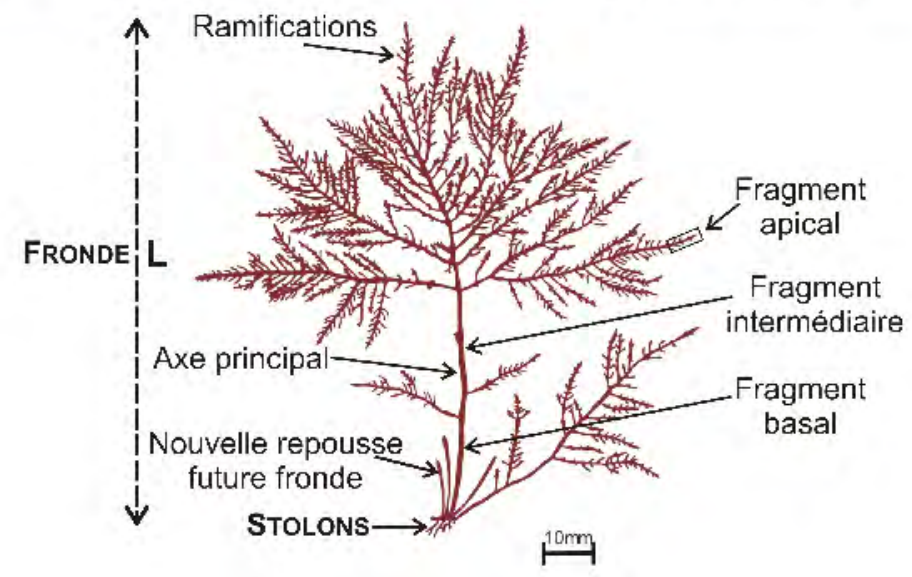

Gelidium sesquipedale

Figure 2 : Morphologie des espèces étudiées.

Le thalle de l'agarophyte Gelidium sesquipedale rouge à rouge brun (Figure 2) présente un aspect robuste et une consistance cartilagineuse. Il est constitué de frondes érigées, regroupées en touffes de taille variable de 10 à $40 \mathrm{~cm}$, s'élevant à partir de filaments rampants (stolons ou rhizoïdes) qui assurent la fixation de l'algue au substrat. La fronde est constituée d'un ensemble d'axes principaux à croissance illimitée porteurs de ramifications latérales à croissance limitée, ce qui donne au thalle une forme pyramidale. Cette espèce forme des peuplements denses sur tous les fonds rocheux en mode battu depuis le niveau des basses mers de vives eaux jusqu'à une profondeur de $20 \mathrm{~m}[24,25]$. G. sesquipedale représente plus de $90 \%$ de la récolte d'algues agarophytes sur les côtes du Maroc. 
Le carraghénophyte Hypnea musciformis est une algue polymorphe de couleur très variable en fonction de l'exposition; tantôt verdâtre, tantôt rouge sombre, formant des touffes de rameaux de consistance ferme, cylindriques et inextricables. Ces rameaux portent des ramifications simples dont les extrémités effilées sont droites ou enroulées en crosse, ce dernier aspect très caractéristique, permet la reconnaissance de cette espèce sans ambiguïté [26] (Figure 2).

\section{2-3. Analyse physicochimique}

Les prélèvements d'eau de mer ont été effectués mensuellement durant les deux années 1996 et 1997. La température de l'eau a été mesurée à l'aide d'un thermomètre à mercure gradué au $1 / 10^{\circ} \mathrm{C}$. La salinité $(\mathrm{S} \%$ ) est déterminée à l'aide d'un réfractomètre portatif. Une courbe d'étalonnage permet d'obtenir directement la salinité en fonction de l'indice de réfraction et de la température.

Les nitrates $\left(\mathrm{NO}_{3}{ }^{2-}\right.$ en $\left.\mathrm{g} . \mathrm{L}^{-1}\right)$ sont réduits quantitativement en nitrites par voie chimique en utilisant le sulfate d'hydrazine [27]. Les nitrites ainsi formés sont dosés par la méthode classique au sulfanilamide/dichlorohydrate décrite par Strickland et Parson [28].

Les phosphates $\left(\mathrm{PO}_{4}{ }^{3-}\right.$ en $\mu$ atg..$\left.^{-1}\right)$ sont dosés par la méthode de Stephens [29], qui est une extension de celle décrite par Murphy et Riley [30]. Elle est très sensible et particulièrement adaptée pour la détermination des phosphates se trouvant en faible concentration en milieu marin.

\section{2-4. Analyse de la croissance}

La mesure des paramètres considérés dans l'évolution de la biologie des trois espèces a été effectuée sur 100 thalles récoltés au hasard sur les gisements naturels, et sont (Figure 2) :

- La longueur du thalle ( $\mathbf{L}$ en $\mathrm{cm}$ ), elle a été déterminée en mesurant la longueur de la fronde érigée avec un double décimètre ;

- La masse de la fronde ( $P$ en $\mathbf{g})$ a été déterminée en pesant chaque thalle à l'aide d'une balance avec une précision au milligramme ;

- Le nombre total de ramifications (RT) est compté pour chaque thalle.

L'ensemble des données a subi un traitement statistique multivarié (logiciel SAS); l'analyse en composantes principales (ACP) qui est un outil de description des tableaux de relevés de mesures comportant $\mathrm{n}$ lignes (prélèvements) et $\mathrm{p}$ colonnes (variables). C'est une méthode d'ordination des relevés ou de description des structures intervariables utilisée lorsque les variables sont quantitatives et transcrites dans des unités diversifiées. La matrice ainsi constituée est formée de 64 relevés mensuels pour les 7 variables. 


\section{Résultats et discussion}

\section{3-1. Analyse physicochimique}

En hiver et début du printemps, la température de l'eau de mer est faible, avec des minima de 12 et $17{ }^{\circ} \mathrm{C}$ en mars 1996 et janvier février 1997 respectivement. Elle augmente progressivement pendant le printemps et l'été pour atteindre des maxima de 24 et $25^{\circ} \mathrm{C}$ en août 1996 et 1997 respectivement.

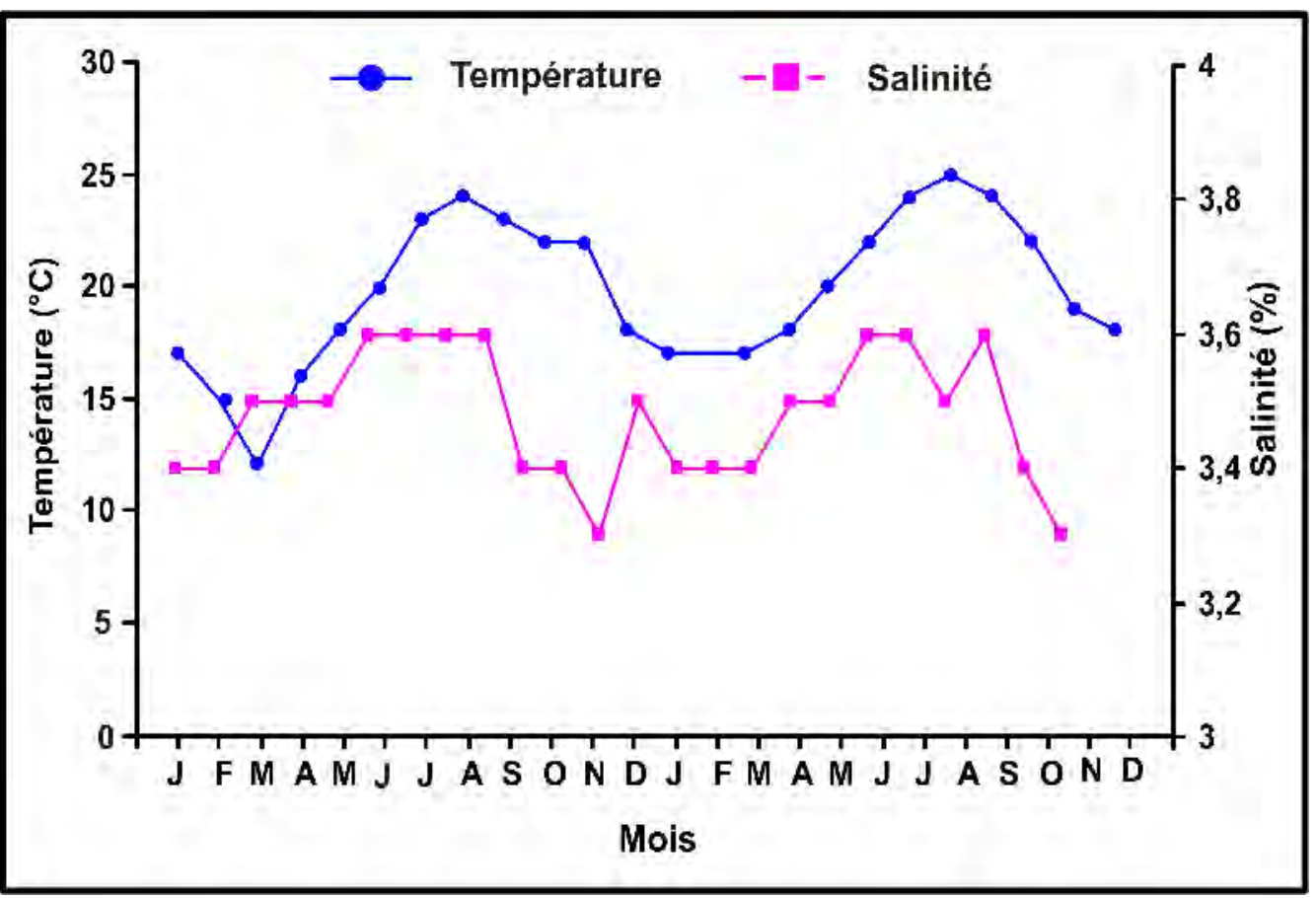

Figure 3 : Variations mensuelles de la température et de la salinité de l'eau de mer à Mehdia (année 1996-1997).

Les salinités les plus faibles sont enregistrées en hiver et en automne (3,4 \% environ). En période estivale la salinité atteint son maximum de 3,6\%. Ces variations peuvent être expliquées par les apports fluviaux au cours des saisons pluvieuses.

Les plus faibles concentrations en nitrates sont notées en fin de printemps - début d'été $\left(0,05\right.$ et $0,5 \mathrm{mg} . \mathrm{L}^{-1}$ en mai-juin 1996 et $0,4 \mathrm{mg} . \mathrm{L}^{-1}$ en avril 1997), un autre minimum est mesuré en automne 1997 (0,35 mg..$^{-1}$ en octobre 1997). Le maximum de concentration en nitrates a été enregistré en octobre $1996\left(2,35 \mathrm{mg} \cdot \mathrm{L}^{-1}\right)$. Les plus faibles concentrations en 
phosphates ont été enregistrées en fin printemps - début été $\left(0,05 \mu\right.$ atg. $L^{-1}$ juin 1996 et juillet 1997). Une valeur maximale de $3 \mu$ atg..$^{-1}$ est notée en avril 1997.

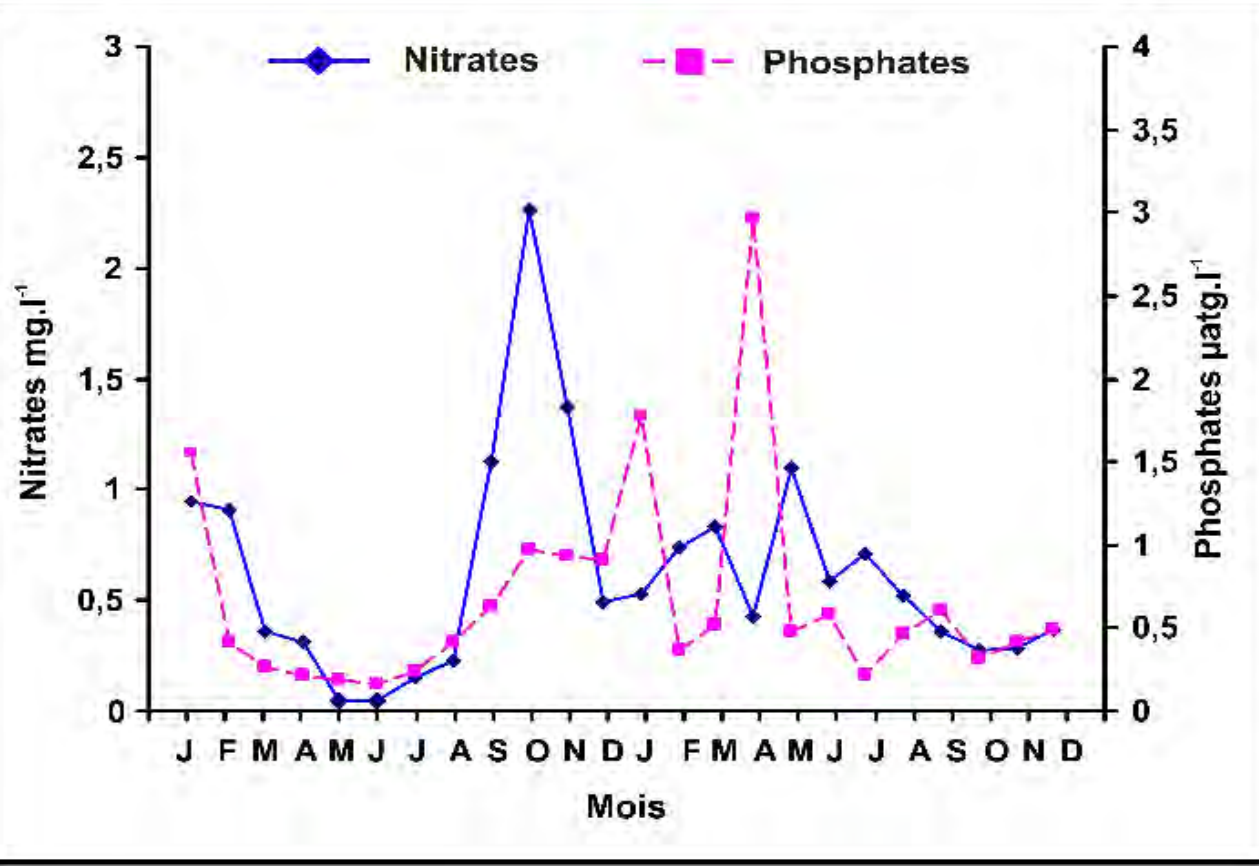

Figure 4 : Variations mensuelles des concentrations en nitrates et en phosphates de l'eau de mer à Mehdia (année 1996-1997).

Les concentrations en nitrates et en phosphates présentent des courbes régulières et superposables en 1996, avec des minima début d'été et fin d'automne et des maxima en automne hiver. La première période correspond à une forte biomasse phytoplanctonique qui épuise les sels nutritifs pour sa prolifération lorsque l'intensité lumineuse et la température augmentent. La deuxième période correspond à une faible activité physiologique des végétaux marins en fin automne et en hiver. Les courbes d'évolution de ces paramètres présentent peu de variations entre 1996 et 1997.

\section{3-2. Analyse de la croissance}

\section{3-2-1. Gracilaria multipartita}

Les périodes de fortes croissance en longueur (Figure 5) sont enregistrées de mars à juillet (avec un maximum de $8,1 \mathrm{~cm}$ en juillet) et en septembre (avec un maximum de 
$11,4 \mathrm{~cm})$. Les longueurs les plus faibles ont été notées en hiver, avec un minimum de $4,1 \mathrm{~cm}$ au cours des mois de janvier à mars.

Le poids des thalles a varié d'une manière similaire. La croissance faible en hiver, subit une légère augmentation entre mars et mai et devient importante à partir de mai pour atteindre un maximum de $1,8 \mathrm{~g}$ en juin. Après une diminution du poids au cours de l'été, un deuxième pic de croissance moins important est enregistré en septembre $(1,1 \mathrm{~g} /$ individu). Par la suite, le poids des thalles diminue pour atteindre le minimum de $0,4 \mathrm{~g}$ /individu en décembre.

La courbe de variation du nombre total de ramifications suit une évolution comparable à celle du poids des thalles. Le nombre moyen de ramifications est minimal entre janvier et mars. Il augmente légèrement entre mars et mai et devient plus important à partir de mai pour atteindre le maximum en juin (114 ramifications par individu), puis chute par la suite pour atteindre un minimum de 30 ramifications par individu en décembre.

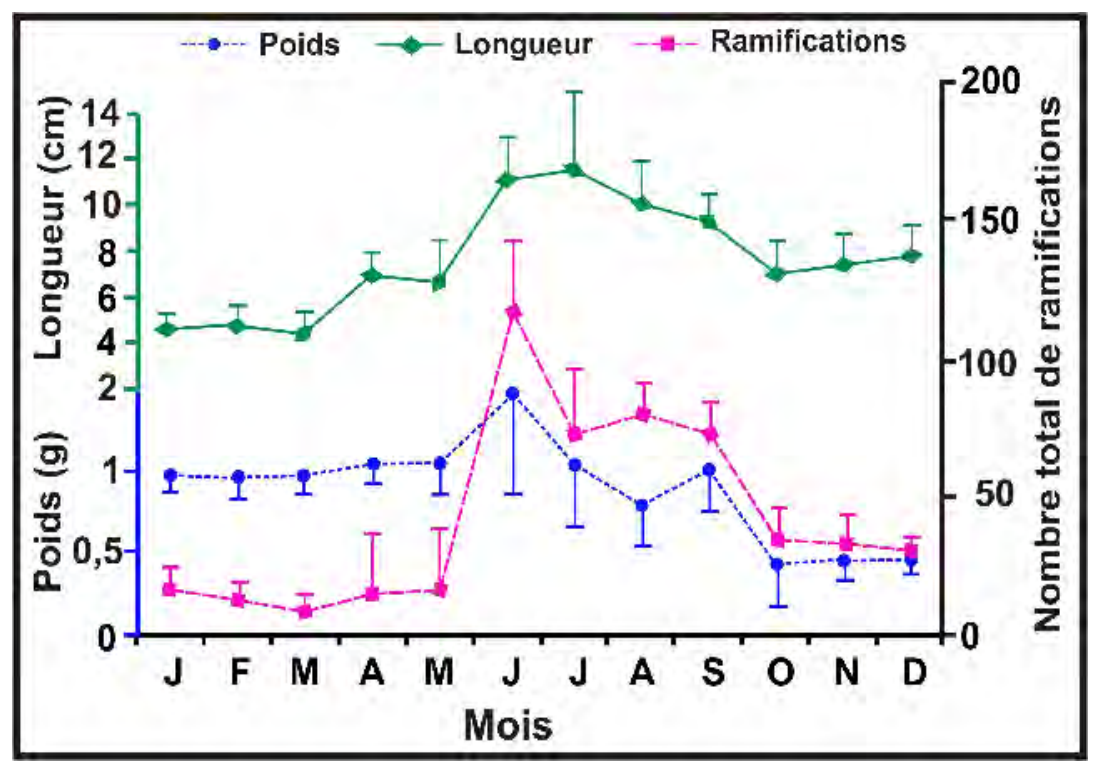

Figure 5 : Variations mensuelles des trois paramètres de croissance (longueur, poids et nombre total de ramifications) de Gracilaria multipartita récolté à Mehdia en 1996 (les barres verticales représentent $\pm \sigma, n=100$ ).

\section{3-2-2. Gelidium sesquipedale}

La longueur moyenne des thalles (Figure 6) augmente progressivement entre janvier et mars, avec un maximum de $9,65 \mathrm{~cm}$ en mars, diminue en avril; puis la croissance reprend de juin à septembre pour atteindre un deuxième maximum de $11 \mathrm{~cm}$ en septembre. Par 
la suite la croissance en longueur diminue pour atteindre un minimum en décembre de 7 cm environ. Les courbes du poids moyen et du nombre de ramification présentent à peu près la même allure, les deux maxima de croissance sont atteints en février puis septembre (0,39- $7 \mathrm{~g}$ /individu et 32-42 ramifications/individu respectivement). II paraît que le gain en poids est plus lié à la ramification qu'à la longueur en janvier et février, les maxima en poids et ramifications sont décalés d'un mois par rapport au maximum en longueur.

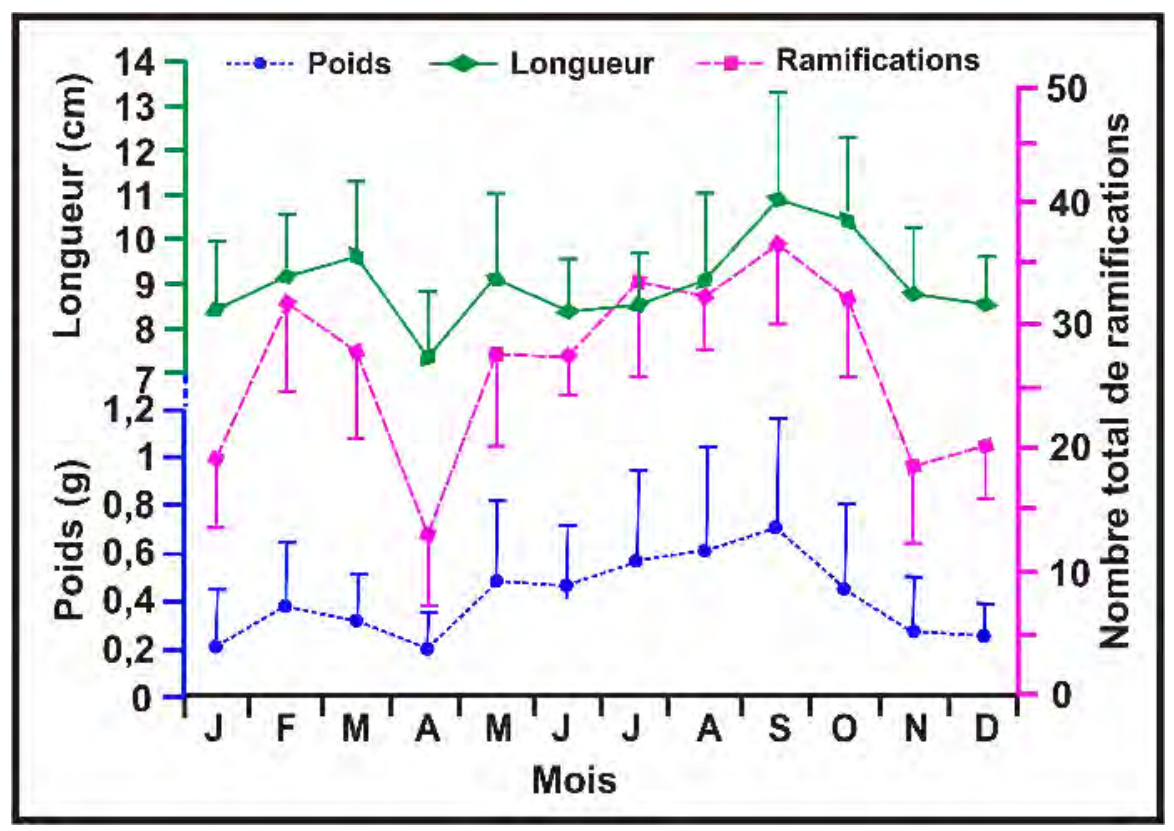

Figure 6 : Variations mensuelles des trois paramètres de croissance (longueur, poids et nombre total de ramifications) de Gelidium sesquipedale récolté à Mehdia en 1996 (les barres verticales représentent $\pm \sigma, n=100$ ).

\section{3-2-3. Hypnea musciformis}

Les courbes de croissance des trois paramètres présentent à peu près la même allure avec deux périodes de croissance plus ou mois actives. La longueur et le poids augmentent de février au mois d'août, puis de septembre à novembre, avec des maxima de 6 et $5,2 \mathrm{~cm}$ pour la longueur et de 0,65 et $0,025 \mathrm{~g} /$ individu respectivement. Le nombre total de ramifications augmente de février à avril puis de septembre à octobre avec des maxima de 8,75 et 7,5 ramifications/individu respectivement. Le nombre total de ramifications augmente entre mai et juin, se stabilise pendant l'été puis diminue à nouveau en septembre. 
Les minima en longueur, en poids et nombre total de ramifications sont enregistrés en février puis en septembre, avec respectivement $4 \mathrm{~cm}, 0,023 \mathrm{~g} /$ individu et 1,2 ramifications/individu puis en $4,5 \mathrm{~cm}, 0,018 \mathrm{~g} /$ individu et 2,75 ramifications/individu. Les valeurs notées au mois de mai sont difficiles à expliquer et peuvent être générées par plusieurs autres facteurs et en particulier à l'hydrodynamisme : cette algue est fragile et dès qu'il y a une forte agitation, l'algue peut se fragmenter et perdre ses ramifications surtout lors de sa fertilité.

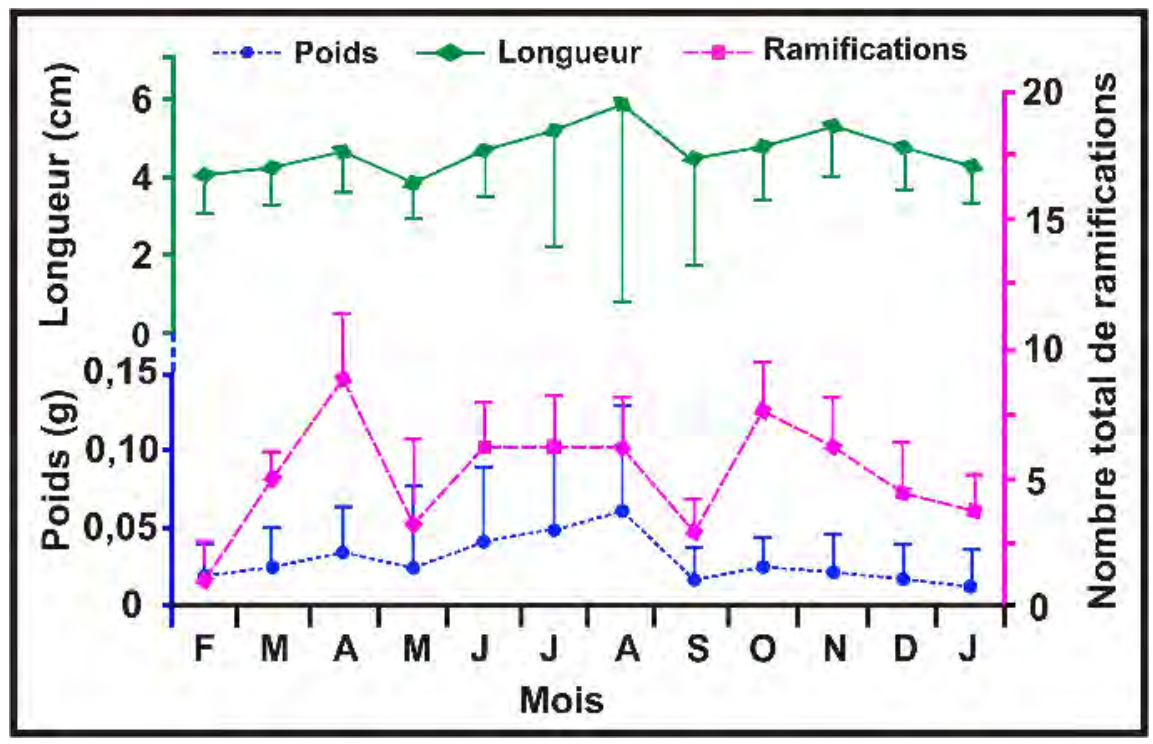

Figure 7 : Variations mensuelles des trois paramètres de croissance (longueur, poids et nombre total de ramifications) d'Hypnea musciformis récolté à Mehdia en 1997 (les barres verticales représentent $\pm \sigma, n=100$ ).

\section{3-2-4. Comparaison du mode de développement des trois espèces par ACP}

La matrice de données de départ est formée par 64 relevés mensuels des espèces considérées au cours desquels les trois paramètres de croissance (L, P et $\mathrm{Rt}$ ) et les quatre facteurs du milieu (température, salinité, nitrates et phosphates) ont été pris en considération (Figure 8).

Les deux premiers axes sont pris en considération pour la description des corrélations entre les variables liées aux structures spatiales qui expliquent $60 \%$ de l'information totale (Figure 8C). Dans le plan factoriel FlxF2, l'axe $\mathrm{Fl}$ contribue à $40 \%$ de l'inertie totale. II dénote l'existence d'un gradient de croissance de droite à gauche défini par les paramètres étudiés; la taille, le poids et le nombre total de ramifications. Les paramètres chimiques du milieu (nitrates et phosphates) paraissent définis 
simultanément par les deux axes $\mathrm{Fl}$ et $\mathrm{F} 2$, alors que la température et la salinité sont gérés uniquement par l'axe factoriel F2 (Figure 8B). Ce dernier contribue à $20 \%$ de l'inertie totale, il définit ainsi un gradient de minéralisation (Figure $8 C$ ).
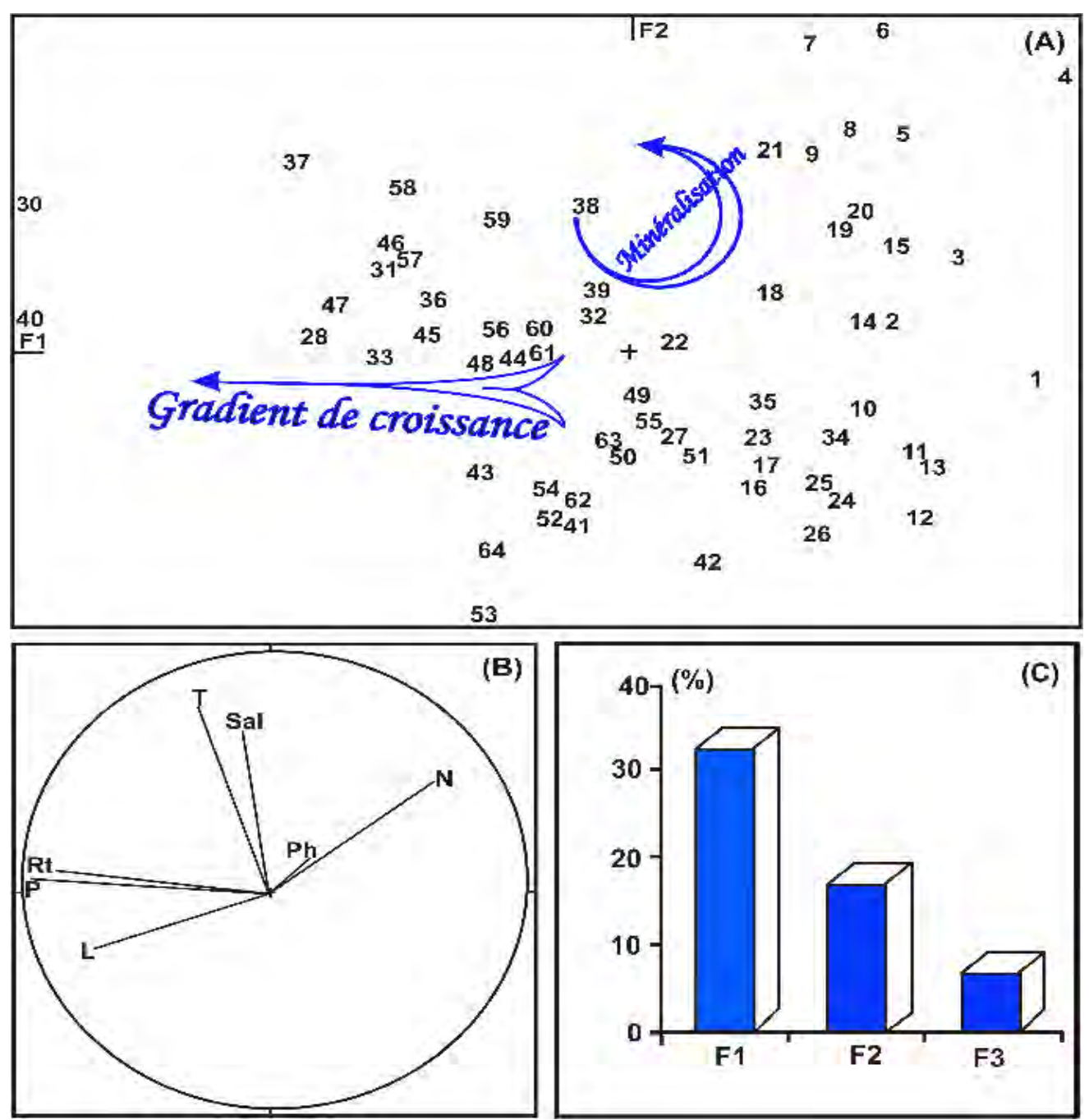

Figure 8 : Représentation graphique dans le plan FIxF2 des relevés des trois espèces étudiées.

A : Carte factorielle des relevés mensuels;

$B$ : Carte factorielle des variables;

$C$ : Répartition de l'inertie entre les axes. 
L'analyse de la carte factorielle présentée par les axes Fl et F2 montre une large répartition des différents prélèvements sur ce plan. Ceci témoigne d'une grande variation de la croissance des trois espèces d'algues considérées au cours de la période d'investigation (Figure 8A). L'évolution des prélèvements mensuels des trois espèces prises séparément est différente d'une algue à l'autre. L'analyse par espèce permet de mieux dégager les affinités, les tendances, les corrélations et d'autres phénomènes intervenant dans la croissance des trois espèces Hypnea musciformis Gracilaria multipartita et de Gelidium sesquipedale.

La projection des relevés mensuels d'Hypnea musciformis sur le plan FlxF2 montre une évolution horizontale et cyclique des prélèvements (Figure 9A-B). Ces derniers sont tous situés négativement par rapport à l'axe $\mathrm{Fl}$. Le cycle biologique de cette espèce semble être géré par les paramètres physiques (température et salinité) et chimiques (nitrates et phosphates) du milieu qui affectent en grande partie le développement et la croissance des thalles de cette espèce (Mouradi et al. soumis dans la revue "I Afrique Science 11 .

L'évolution des prélèvements de Gracilaria multipartita projetés sur la carte factorielle définie par l'axe FlxF2 montre qu'au cours des mois d'été et d'une partie de l'automne où la température et la minéralisation du milieu sont importantes, la croissance de l'espèce est forte [20]. Cependant, les mois d'hiver et de printemps sont caractérisés par une croissance moins marquée que lors des mois précédents (Figure 9C-D).

Les prélèvements mensuels du Gelidium sesquipedale projetés sur le plan factoriel FlxF2 montrent une évolution horizontale et cyclique le long de l'axe F2. Les facteurs environnementaux semblent peu affecter le cycle biologique de cette espèce. La croissance est plus importante au cours de l'été (juin-juillet - aô̂t) et pendant une partie de l'automne (septembre-octobre) caractérisés par des températures et des intensités lumineuses moyennes (Figure 9E-F).

L'analyse globale de la carte factorielle montre une large répartition des prélèvements mensuels qui montrent des différences spécifiques dans les rythmes de croissance des trois espèces étudiées. Ainsi, Gracilaria multipartita est l'espèce qui présente un développement important des paramètres de croissance surtout pendant la période estivale. Gelidium sesquipedale et Hypnea musciformis présentent une évolution cyclique de la croissance, importante pendant l'été et une partie de l'automne et faible en hiver et au printemps. 

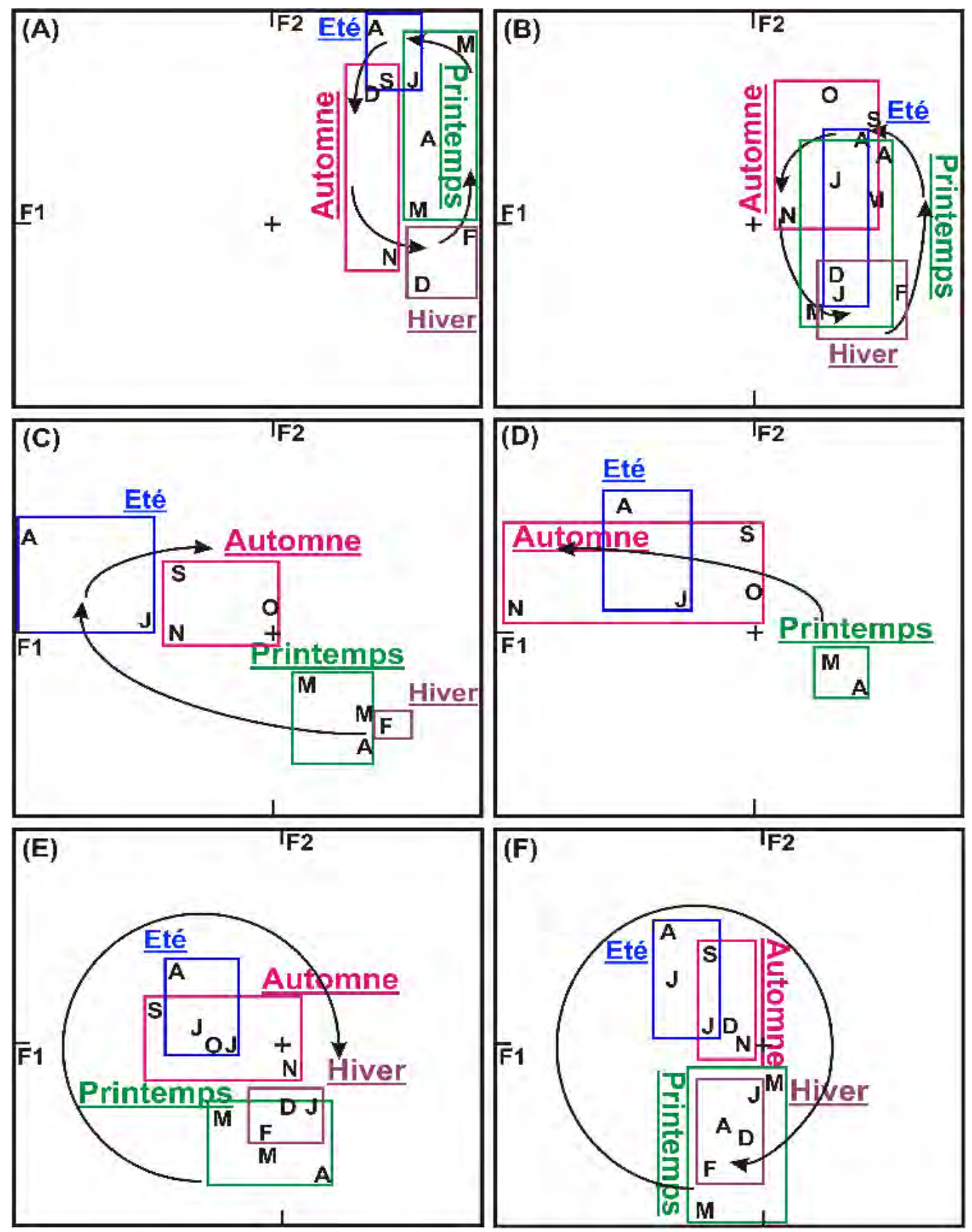

Figure 9 : Projection des différents prélèvements sur le plan factoriel FlxF2.

$A-B$ : Relevés mensuels d'Hypnea musciformis ;

$C-D:$ Relevés mensuels de Gracilaria multipartita ;

E-F : Relevés mensuels de Gelidium sesquipedale. 


\section{Discussion}

Le développement des thalles des algues est l'expression du code génétique, modulé par divers facteurs externes (lumière, température, composition du milieu, ...) et internes (échanges intercellulaires, régulations : métaboliques, hormonales, ...) [31-33]. La lumière agit notamment sur la polarité, le rythme de division, l'allongement cellulaire, la différenciation des organes reproducteurs et sur les photorécepteurs [34]. La température est considérée comme le facteur de contrôle principal de la distribution géographique des algues [35] et le facteur dominant dans les phénomènes saisonniers de croissance et de reproduction [36,37]. Le phosphore, et plus spécialement l'azote, sont normalement présents dans l'eau de mer à des concentrations si faibles qu'ils deviennent parfois des facteurs nutritifs limitants [38,39]. Si l'action de ces facteurs est généralement connue, chaque espèce réagit différemment aux variations de ces facteurs et à chaque espèce correspond un cycle de développement caractéristique.

Dans cette étude, nous comparons le cycle de développement de trois espèces abondantes au sein d'un même site de récolte, et économiquement importantes pour le Maroc. En effet, même si ces trois espèces vivent dans la même station, Gelidium sesquipedale se trouve principalement en infralittoral et en mode battu, c'est une espèce pérennante. Gracilaria multipartita une autre agarophyte que nous rencontrons principalement en zone semi battu; c'est une algue dont les frondes sont annuelles. Hypnea musciformis est une algue polymorphe de couleur très variable en fonction de l'exposition ; tantôt verdâtre, tantôt rouge sombre, qui préfère les zones plus calmes [40]. Les trois espèces appartiennent au branchement des rhodophycées caractérisées par un cycle trigénétique haplodiplophasique.

L'étude du cycle de développement des trois espèces présentent un certain nombre de similitude, mais diffère dans les détails (gamme de tolérance de chaque facteur, périodes de fructification, ...). Ainsi, les trois espèces présentent deux périodes de croissance active, la première est légèrement décalée dans le temps d'une espèce à l'autre, alors que la deuxième, en automne est la même pour les trois espèces.

G. multipartita présentent une croissance faible ou nulle en hiver et une croissance active au printemps-été et en automne, des résultats similaires concernant les périodes de croissance ont été obtenus par [41]. A partir de septembre-octobre les frondes se fragmentent et disparaissent et les individus passent l'hiver sous forme de disque. Pondevida et al., [42] ont montré que la biomasse de $G$. cervicornis au Brésil présente un maximum de croissance pendant l'été et un minimum en hiver. Les mêmes résultats ont été notés par Luhan [43] pour $G$. heteroclada et $G$. baillinae. Lee et al. [44] ont distingué chez $G$. tenusitipitata une forme d'été avec des thalles développés et une forme d'hiver très réduite. Les travaux d'El Bacha et al. [20] se sont intéressés à l'influence des 
facteurs environnementaux, pris séparément, sur la croissance de $G$. multipartia en culture et ont montré que cette espèce présente un taux de croissance avoisinant $2,5 \% . j^{-1}$. L'espèce présente un taux de croissance maximum pour des intensités lumineuses comprises entre 100 et $300 \mu \mathrm{mol} . \mathrm{m}^{-2} \mathrm{~s}^{-1}$ avec une lumière blanche, une concentration en phosphate inférieure à $5 \mathrm{mM}$, une concentration en carbonate inférieure à $5 \mathrm{mM}$, une salinité comprise entre 24 et $46 \mathrm{ppt}$, l'algue assimile mieux l'azote sous forme d'ammonium avec une concentration entre 0,5 et $4 \mathrm{mM}$. Ce taux de croissance peut être amélioré en étudiant les interactions entre ces facteurs.

Généralement, la croissance optimale des gracilaires est notée pour des températures comprises entre 24 et $30^{\circ} \mathrm{C}$ [45]. Lapointe et Ryther [46] ont montré que pour des températures au-dessous de $12{ }^{\circ} \mathrm{C}$, les gracilaires peuvent survivre, sans pour autant crô̂tre. La salinité enregistrée au cours de la période d'étude est peu variable. Toutefois, les Gracilaires sont très plastiques dans leurs réponses aux variations de la salinité du fait quelles sont des espèces euryhalines [47]. Les deux facteurs température et lumière ont un effet important sur la croissance des gracilaires. D'après Friedlander et al. [48] la vitesse de croissance est hautement dépendante de la température chez $G$. tikvahiae et d'après Lapointe [49], la croissance de $G$. multipartita est dépendante de l'intensité lumineuse. Cet effet de la lumière a été également constaté chez $G$. verrucosa [50].

G. sesquipedale présente une croissance active entre mai et juillet et une deuxième entre septembre et octobre ; la croissance est faible entre Janvier et avril et négative entre novembre et janvier. Des résultats similaires ont été obtenus par Mouradi et al. en 1992 et en 1993 [51,52] sur $G$. Iatifolium récolté à Roscoff (France); cependant $G$. latifolium présente une plus grande plasticité morphologique que sur $G$. sesquipedale récolté au Maroc en 1999 [53]. Le rythme d'allongement de $G$. sesquipedale est faible $(9 \mathrm{~cm} / \mathrm{an})$. Des valeurs du même ordre ont été trouvées pour d'autres espèces de Gelidium $[31,53,54]$. La croissance lente de ces espèces a été reliée à des facteurs intrinsèques, plutôt qu'un trait qui peut être modifié par les facteurs externes $[54,55]$.

Le suivi de l'évolution des paramètres de croissance de $G$. sesquipedale a permis de montrer l'hétérogénéité des populations de cette algue même dans les zones non exploitées et de distinguer des périodes de croissance et de régénération dans le développement des thalles. Seoane-Comba [56] a montré que la croissance de $G$. Sesquipedale est faible car elle est liée à des facteurs intrinsèques, ce qui donne une forme régulière et pyramidale à cette espèce tout le long de l'année en milieu naturel au niveau de l'infralittoral; en effet, nous n'avons pas noté une grande variation morphologique de cette espèce récoltée au niveau de l'infralittoral. Cependant les travaux de Givernaud et al. en 2003 [22], ont montré que les facteurs externes peuvent changer la morphologie de ce dernier; en effet la culture de cette espèce sans substrat ou sous un stress thermique donne des thalles peu ramifiés, effilés rappelant la 
morphologie des stolons de l'espèce. Ceux de Sousa-Pinto et al. [57] ont montré l'influence de la lumière sur la qualité et la quantité de l'agar produit par cette espèce. Cette espèce a montré une très forte capacité de régénération, une faible variation morphologique et une grande hétérogènéité des populations naturelles [58].

En fin septembre, les frondes se dégradent suite à la libération des cellules reproductrices ou à une fragmentation naturelle desfrondes par la houle. Pendant cette période le nombre de thalles récoltés en échouage est très important. Le même résultat a été signalé par Kaas et Barabaroux [59] dans les populations de $G$. sesquipedale du sud de la France.

La période correspondant à un gain en poids s'étale de mai à septembre et est liée à une augmentation de la longueur et du nombre de ramifications. Si la période de septembre à janvier correspond à une phase de vieillissement des frondes qui se termine en janvier avec une détérioration des extrémités des thalles, la période printanière, correspond à la période de forte régénération de $G$. sesquipedale. Les fragments, qui se détachent du thalle, se fixent sur le substrat et donnent de nouvelles repousses qui formeront de nouvelles frondes. Ce phénomène de forte capacité de régénération de l'algue a été utilisé par plusieurs auteurs $[18,22,60,61]$ pour essayer de cultiver ou de réensemencer les champs de $G$. sesquipedale.

Le suivi du cycle biologique d'Hypnea musciformis en milieu naturel montre également deux périodes de croissance active : la première en été (mai-août) et la deuxième de moindre importance en automne. Des résultats similaires ont été obtenus par d'autres auteurs sur la même espèce récoltée en Tanzanie [40]. Ces auteurs ont montré que cette espèce se développe bien dans des milieux calmes quand l'intensité lumineuse et les concentrations en phosphate et en nitrates sont élevées. Guist et al. [62] ont montré que le taux de croissance d' $H$. musciformis est important et peut atteindre $20 \% / i^{-1}$ lorsque la température de l'eau de mer est comprise entre 18 et $24^{\circ} \mathrm{C}$ couplé à une agitation en flux continu et à un supplément en nitrates et phosphates. Cette vitesse de croissance serait supérieure à celle obtenue pour Eukeuma denticulatum ou kappaphycus alvarezii cultivés aux philippines et qui constituent les principales espèces pour la production de carraghénane à l'échelle mondiale [63]; ce qui veut dire que cette espèce peut constituer un potentiel économique important pour le Maroc.

La croissance d'H. musciformis montre des fluctuations dues aux variations des facteurs environnementaux telle que la salinité, la température et la dessiccation prolongée [64], l'intensité lumineuse [65], l'humidité de l'air [66], l'adaptation au substrat [67]. Le principal facteur contrôlant la production saisonnière d'Hypnea musciformis en culture est également le mouvement de l'eau [68].

L'analyse quantitative par les différentes méthodes statistiques a permis de dégager les principales affinités de croissance de chaque espèce et viennent confirmer les 
observations morphologiques notées au cours d'un cycle annuel. Le cycle de développement d' $H$. musciformis et de $G$. multipartita est plus influencé par les facteurs externes que celui de $G$. sesquipedale, en effet, comme cela a été montré par d'autres auteurs cette espèce est très difficile à maintenir en culture $[24,58,69,70]$; il apparâ̂t que c'est une caractéristique de ce genre qui se trouve naturellement en mode battu.

L'analyse globale de la carte factorielle montre une large répartition des prélèvements mensuels qui montrent des différences spécifiques dans les rythmes de croissance des trois espèces étudiées. Ainsi, Gracilaria multipartita est l'espèce qui présente le développement le plus important des paramètres de croissance surtout pendant la période estivale [71]. Ces résultats concordent avec ceux obtenus par Yakovleva et al. en 2001 [13] et par Bulboa et Macchiavello la même année [15], qui ont montré que les Gigartinales et les Gracilariales présentent une croissance optimale à des températures estivales comprises entre 24 et $30^{\circ} \mathrm{C}$.

Le cycle biologique d'Hypnea musciformis semble être affecté par un gradient de minéralisation qui lui impose un rythme de croissance limité par rapport aux deux autres espèces d'algues rouges. Des résultats similaires ont été obtenus par Guist et al. [62], les meilleurs taux de croissance sont obtenus sous de fortes concentration nitrates et phosphates, or ces derniers se trouvent en faible concentrations en milieu marin. Pour le développement de l'aquaculture de cette espèce, il est nécessaire de fournir régulièrement des sels nutritifs.

Les périodes de croissance active de Gracilaria multipartita sont caractérisées par des températures et des intensités lumineuses plus importantes et par des teneurs élevées en nitrates et en phosphates [72,73]. Molloy et Bolton [74] ont montré que la production de biomasse des espèces de Gracilaria est plus importante en été qu'en hiver et ont attribué cette différence à l'augmentation importante en été de l'intensité lumineuse.

\section{Conclusion}

Au terme de cette étude, il apparaît que les rythmes de croissance des trois algues rouges sont différents et seraient liés en plus de leur physiologie propre, à l'influence des facteurs environnementaux.

Il est essentiel de noter que chez les trois espèces, il n'y a pas une succession saisonnière de la phase haplöide (Gamétophytes) et de la phase diploïde (tétrasporophyte). Cette situation n'est pas rare chez de nombreuses Rhodophycées, les deux phases se déroulent simultanément. Les trois espèces présentent deux phases actives : la première en printemps pour Gracilaria multipartita, en été pour Hypnea 
musciformis et s'étale depuis mai jusqu'en septembre pour Gelidium sesquipedale. La deuxième est en automne pour les trois espèces.

La croissance de Gracilaria multipartita et de Hypnea musciformis est dépendante des facteurs environnementaux, les deux espèces présentent une variation de leur morphologie et présentent des gammes de tolérance assez larges, ces deux espèces peuvent atteindre des taux de croissance élevés en culture et méritent d'être cultivées au Maroc pour une production industrielle. Gelidium sesquipedale présente un rythme de croissance faible liée à des facteurs intrinsèques de l'espèce. II vit dans des milieux fortement agités; sa culture est donc difficile à réaliser. Pour l'industrie locale, il est nécessaire d'établir un programme de gestion des stocks naturels et d'assurer le repeuplement des champs surexploités.

La biomasse de Gracilaria multipartita est maximale à la fin du mois de juin, celle d'Hypnea musciformis est maximale en août. L'exploitation de ces espèces devrait être réalisée dès ce moment avec une période de récolte réduite à deux mois au maximum pour laisser l'algue croître de nouveau en automne. Les populations de $G$. sesquipedale présentent une grande hétérogénéité, l'exploitation de l'espèce doit se faire avec précaution au cours de l'été, mais en respectant les systèmes de fixation de l'espèce et en ne récoltant que les thalles les plus âgés.

\section{Remerciements}

Ces travaux ont été réalisés dans le cadre de la coopération franco-marocaine (Al : 94/768, Al: II0/SVS/97, AI MA/01/20 et AI MA/06/ 154) et de la coopération avec la société locale Setexam, que nous tenons à remercier vivement. Nos remerciements vont également au CRNTS et au ministère de l'Education, de l'Enseignement Supérieur, de la Formation des Cadres et de la Recherche Scientifique pour leur soutien financier.

\section{Références}

[1] - FAO-Fisheries Department, "Fishery Information, Data and Statistics Unit. Fishstat Plus. Universal Software for fishery statistical time series". Version 2.3. last updated March 2004. Food and Agriculture Organisation. United Nations. Rome. Italy (2004).

[2] - D. J. McHugh. "A Guide to the Seaweed Industry". Food and Agriculture Organisation. United Nations. Fisheries Technical Paper 441, Rome, Italy. (2003) 123 p.

[3] - J. S. Craigie. "Cell walls". In K.M. Sheath and R. G. (eds). Biology of the red algae. Cambridge University Press (1990) 221-259. 
[4] - I. A. Abbott. "Ethnobotany of seaweeds: clues to uses of seaweeds". Hydrobiologia, 326/327 (1996) 15-20.

[5] - M. S. Doty. "Betaphycus philippinensis gen. et sp. nov- and related species (Solieriaceae, Gigartinales)." In Abbott (ed.), Taxonomy of economic seaweeds with reference to some Pacific specie. La Jolla: California Sea Grant College Program [Report T-CSGCP-035]. 5 (1995) 237-245.

[6]- A. Jensen. "Present and future needs for algae and algal products". Hydrobiologia 260/261 (1993) 15-23.

[7] - R. Perez. "Ces algues qui nous entourent, conception actuelle, rôle dans la biosphère, utilisations, culture". Ifremer. (1997) 272p.

[8] - M. J. Carlucci, C. A. Pujol, M. Ciancia, M. D. Noseda, M. C. Matulewicz, E. B. Damonte and A. S. Cerezo. "Antiherpetic and anticoagulant properties of carrageenans from the red seaweed Gigarlina skottsbergii and their cyclized derivatives: correlation between structure and biological activity". Inter. J. Biolog. Macromol. 20 (1997) 97-105.

[9] - W. R. L. Farias, A. P. Valenta, M. S. Peraira and P. A. S. Mourâo. "Structure and anticoagulant activity of sulfates galagtans". J. Biolog. Chem. 22 (2000) 29299 29307.

[10] - G. S. B. Viana, A. L. P. Freitas, M. M. L. Lima, L. A. P. Vieira, M. C. H. Andrade and N. M. B. Benevides. "Antinociceptive activity of sulfated carbohydrates from the red algae Bryothamnion seaforthii (Thrner) Kütz. and B. triquetrum (S.G. Gmel.) M. Howe". Braz. J. Medical and Biolog. Resear. 35 (6) (2002) 713-722.

[11] - M. Indergaard. "The aquatic ressource". In W. A. Cote (ed). Biomass utilisation. Plenum Press, New York. (1983) 137-168.

[12] - T. Chopin, M. D. Hanisak and J. S. Craigie. "Carrageenan from Kallymenia westii (Rhodophyceae) with a review of the phycocolloids produced by the Cryptonemiales". Bot. Mar. 37 (1994) 433-444.

[13] - M. Yakovleva, I. M. Yermak, E. A. Titlyanov, A. O. Barabanova, V. P. Glazunov and A. V. Skriptsova. "Changes in growth rate, anatomy and polysaccharide content of a sterile form of Tichocarpus crinatus (Gmel.) Rupr. (Rhodophyta, Tichocarpaceae) grown under differing photon irradiances in the Sea of Japan, Russia". Bot. Mar. 44 (2001) 493-500.

[14] - J. Pfetzing, D. B. Stengel, M. M. Cuffe, A. V. Savage and M. D. Guiry. "Effects of temperature and prolonged emersion on photosynthesis, carbohydrate content and growth of the brown intertidal alga Pelvetia canaliculata". Bot. Mar. 43 (2000) 399-407.

[15] - C. R. Bulboa and J. E. Macchiavello. "The effects of light and temperature on different phases of the life cycle in the carrageenan producing alga Chondracanthus chamissoi (Rhodophyta, Gigartinales)'. Bot. Mar. 44 (2001) 371-374. 
[16] - A. H. Buschmann, D. Varela, M. Cifuentes, M. D. C. Hernandez-Gonzalez, L. Henriquez, R. Westermeier and J. A. Correa. "Experimental indoor cultivation of the carrageenophytic red alga Gigartina skottsbergii". Aquaculture. 241 (2004) $357-370$.

[17]- M. L. Quartino, H. E. Zaixso and A. L. Boraso De Zaixso. "Biological and environmental characterization of marine macroalgal assemblages in Potter Cove, South Shetland Islands, Antarctica". Bot. Mar. 48 (2005) 187-197.

[18] - J. Silva and R. Santos. "Comparative ecophysiology of Gelidium sesquipedale (Rhodophyta). Erect fronds and prostat system". Proceeding of the 17th international seaweed symposium Cape town, A. R. 0. Chapman, R. J. Anderson, V. Vreeland and T. R. Davison (eds), Oxford University press (2003) 417-424 p.

[19] - S. El Bacha, A. Mouradi, A. El Gourii, B. Benazzouz and T. Givernaud. "Biologial cycle of the agarophyte Gracilaria multipartita (Clemente) Harvey (Rhodophyceae, Gracilariales) on the Moroccan atlantic coast". Actes Inst. Agron. Vet. Maroc. 24 (1-2) (2004a) 23-34.

[20] - S. El Bacha, A. El Gourii, Th. Givernaud, Y. Lemoine and A. Mouradi. "Ecophysiological of the agarophyte Gracilaria multipartita (Clemente) Harvey (Rhodophyceae, Gracilariales)". Actes Inst. Agron. Vet. Maroc. 24 (3-4) (2004b).95103.

[21] - Th. Givernaud, N. Sqali, 0. Barbaroux, A. Orbi, Y. Semmaoui, N. Rezzoum, A. Mouradi and R. Kaas. "Mapping and biomass estimation for a harvested population of Gelidium sesquipedale (Turn.) Thuret (Rhodophyta, Gelidiales) along the Atlantic coast of Moroco". Phycologia. 44 (1) (2005) 66-71.

[22] - Th. Givernaud, A. Mouradi, L.M. Hassani, R. Akallal and J. Riyahi. "Design of a new technique for reseeding of over harvested bed of Gelidium sesquipedale (Turn.) Thuret (Rhodophyta, Gelidiales) in Morocco". Proceeding of the 17th international seaweed symposium Cap town, A. R. 0. Chapman, R. J. Anderson, V. Vreeland and T. R. Davison (eds), Oxford University press (2003) 123-130.

[23] - L. J. Goff, D. A. Moon and A. W. Coleman. "Molecular delineation of species and species relationships in the red algal agarophytes Gracilariopsis and Gracilaria (Gracilariales)". J. Phycol. 30 (1994) 521-537.

[24] - I. Kabbaj. "Biologie de Gelidium sesquipedale des côtes marocaines: étude des peuplements, écophysiologie, cytologie et morphogenèse". Thèse de Doctorat d'Université, Univ. de Caen, France. (1994) 126 p.

[25] - S. Benhissoune, C. E. Boudouresque, M.P. Boudouresque and Verlaque. "A checklist of the seaweeds of the Mediterranean and Atlantic coasts of Morocco. III Rhodophyceae (excluding Ceramiales)". Bot.Mar. 45 (2002) 391-412.

[26] - P. Gayral. "Les algues de la côte Atlantique marocaine". Soc. Sc. Nat. et Phys. du Maroc. Rabat. (1958) 527p. 
[27] - J. B. Mullin and J. P. Riley. "The spectrophotometric determination of silicatesilicon in natural waters with special reference to seawater". Anal. Chim. Acta. 12 (1955) 162-170.

[28] - J. D. H. Strickland and T. R. Parson. "A practical handbook of seawater analysis". Bull. Fish. Res. Bd Can. 167 (1972) 71-89.

[29] - E. Stephens. "A practical handbook of seawater analysis". Limnol. Ocean. 8 (1963) $361 \mathrm{p}$.

[30] - J. Murphy and J.P. Rilley. "A modified single solution method for determination of phosphate in natural waters". Anal. Chem. Aca. 26 (1962) 31-36.

[31] - D. C. Barilotti. "Genetic consideration and experimental design of outplanting studies". in I.A. Abbot, M. S. Foster and L. Eklund (eds) Pacific seaweed aquaculture, La Jolla. Univ. of California. (1980)10-18.

[32] - C. Perrone and G.P. Felicini. "Dominance apicale et morphogénèse chez Petroglossum nicaeense (Duby) Schotter (Rhodophyceae). Phycologia, 13 (3) (1974) 187-194.

[33] - Perrone, C. and Felicini, G. P. "Polarité dans la fronde de Schottera nicaeensis (Phyllophoracées)". Phycologia. 20 (2) (1981) 142-146.

[34] - K. Luning. "Seaweeds: their environnement, biogeography and ecophysiology". Wiley and Sons (eds), New-York. (1990) 527 p.

[35] - B. Santelices. "Synopsis of biological data on the seaweed genera Gelidium and Pterocladia (Rhodophyta)". - FA0 Fish. Synop. 145 (1988) 55 p.

[36] - J. A. Seoane-Camba. "Crecimimento, production y desprendimiento de biomassa en Gelidium sesquipedale (Clem.) Thuret". Proc. 6th. Int. seaweed Symp.(1969) 365-374.

[37] - D. C. Barilotti and W. Silverhorne. "A resource management study of Gelidium robustym". $7^{\text {th }}$. Proc. Int. Seaweed Symp. (1972) 255-261.

[38] - J. A. Deboer. "Nutrients". In C. S. Lobban, and M. J. Wynne (eds). The biology of seaweeds, Blackwell, Oxford. (1981) 356-392.

[39] - C. S. Lobban and P. J. Harrison. "Seaweed ecology and physiology". Cambridge University Press, New York. (1994) 366p.

[40] - M. S. P. Mtolera, J. Collén, M. Pedersén and A. Semesi. "Destructive hydrogen peroxide production in Eucheuma denticulatum (Rhodophyta) during stress caused by elevated pH, high light intensities and competition with other species". Eur. J. Phycol. 30 (1995) 289-297.

[41] - R. C. Engel-Palmiter. "Fonctionnement des populations d'une espèce d'algue rouge marine haplo-diplophasique: structures, dynamiques démographiques ef génétiques chez Gracilaria gracilis". Thèse de Doctorat en sciences, Université de Paris XI Orsay. (2000) 185p. 
[42] - H. B. Pondevida and A. Q. Hurtado-Ponce. "Assessment of some agarophytes from the coastal Iloilo, Philippines. I. Seasonnal variation in the biomass of Gracilaria changii, G. manilaensis and G. bailinae (Gracilariales, Rhodophyta)". Bot. Mar. 39 (1996) 117-122.

[43] - M. R. J. Luhan. "Biomass and reproductive states of Gracilaria heteroclada Zhang et Xia collected from Jaro, Central Philippines". Bot. Mar. 39 (1996) 207-211.

[44] - T. M. Lee, Y. C. Chang and Y. H. LIN. "Differences in physiological responses between winter and summer Gracilaria tenvistipitata (Gracilariales, Rhodophyta) to varying temperature". Bot. Bull. Acad. Sin. 49 (1999) 93-100.

[45] - D. Hanisak. "Cultivation of Gracilaria and other macroalgae in Florida for energy production. In: BIRD, K., and BENSON, P. Eds. Seaweed cultivation for renewable resources". Elsevier, Amsterdam. (1987) 191-218.

[46] - B. E. Lapointe and J. H. Ryther. "The effect of nitrogen and seawater rate on the growth and biochemical composition of Gracilaria foliifera var. angustissima in mass outdoor cultures". Bot. Mar. 22 (1979) 527-339.

[47] - C. P. Dawes. "Suspended cultivation of Gracilaria in the sea". J. Appl. Phycol. 7 (1995) 303-313.

[48] - M. Friedlander, R. Shalev, T. Ganor, S. Strimling, A. Ben-Amotz, H. Klar, and Y. Wax. "Seasonnal fluctuations of growth rate and chemical composition of Gracilaria conferta in outdoor culture in Israef'. Hydrobiologia. 151/152 (1987) 501-507.

[49] - B. E. Lapointe. "Phosphorus and nitrogen limited photosynthesis and growth of Gracilaria tikvahiae (Rhodophyceae) in the Florida Keys: an experimental study". Marine Biology and ecology, 93 (1987) 211-222.

[50] - J. N. C. Whyte, J. R. Englar, R. G. Saunders and J. C. Lindsay. "Seasonal variations in the biomass, quantity and quality of agar from the reproductive and vegetative stages of Gracilaria verrucosa type". Bot. Mar. 24 (1981) 493-501.

[51] - A. Mouradi-Givernaud, T. Givernaud, H. Morvan and J. Cosson. "Agar from Gelidium latifolium (Rhodophyceae, Gélidiales): Biochemical composition and seasonal variations". Bot. Mar. 35 (1992) 153-159.

[52] - A. Mouradi-Givernaud, Th. Givernaud, H. Morvan, and J. Cosson. "Annual variations of biochemical composition of Gelidium latifolium (Greville) Thuret et Bornet". Hydrobiologia. 260/261 (1993) 607-612.

[53] - A. Mouradi-Givernaud, L.M. Hassani, T. Givernaud, Y. Lemoine and 0. Benharbet. "Biology and agar composition of Gelidium sesquipedale harvested along the atlantic coast of Morocco". Hydrobiologia. 398/399 (1999) 391-395.

[54] - J.G. Stewart. "Vegetative growth rates of Pterocladia capillacea (Gelidiaceae, Rhodophyta)". Bot. Mar. 27 (1984) 85-94. 
[55] - A. Mouradi. "Recherches biologiques et biochimiques pour la production d'agarose chez Gelidium latifolium". Thèse de Doctorat ès Sciences Naturelles, Université de Caen, France (1992) 315 p.

[56] - J. A. Seoane-Comba. "Algunos datos de intrés en la recoleccion de Gelidium sesquipedale". Publ. Tec. Junta Estud. Pesca, Madr. 5 (1966) 437-455.

[57] - I. Sousa-Pinto, E. Murano, S. Coelho, A. Felga and R. Pereira. "The effect of light on growth and agar content of Gelidium pulchellum (Gelidiaceae, Rhodophyta) in culture". Hydrobiologia, 398/399 (1999) 329-338.

[58] - L. M. Hassani. "Biologie, biochimie et écophysiologie de l'agarophyte Gelidium sesquipedale (Turner) Thuret (Rhodophycées, Gélidiales) II. Thése de Doctorat Université Ibn Tofail, Fac. Sci., Kénitra, Maroc. (2000)156 pp.

[59] - R. Kaas and 0. Barabaroux. "Exploitation de la ressource en algue rouge Gelidium sesquipedale de la Bidassoa à l'Adour : Etude de l'impact d'une récolte industrielle par plongeur sur la ressource et sur les autres formes actuelles de ramassage". $10^{\text {ème }}$ rencontres interrégionales de l'AGLIA, Saint Jean de Luz, 20/21 novembrel997 (1998) $71-75$.

[60] - J. M. Salinas. "Spray systeme for re-attachement of Gelidium sesquipedale (Clem.) Born. and Thur. (Gelidiales : Rhodophyta)". Hydrobiologia. 221 (1991) 107117.

[61] - A. Borja. "Impacto de la cosecha y recuperation de la biomassa del alga Gelidium sesquipedale sometida a dos formas de exploitacion en el Pais Vasco (Espana)". Aquat. Living Resour. 7 (1994) 59-66.

[62] - G. G. Jr. Guist, C. J. Dawes and J. R. Castle. "Mariculture of the red seaweed, Hypnea musciformis". Aquculture 28 (1982) 375-384.

[63] - A. Buriyo, M. Mtolera and A. Semesi. "The effect of seasons on yield and quality of carrageenan from Tanzanian red alga Eucheuma denticulatum (Gigartinales, Rhodophyta)". S. Afr. J. Bot. 67 (2001) 488-491.

[64] - K. E. Mshigeni and W.R. Mziray. "Studies on the littoral ecology and ecophysiology of the carrageenophytes, Hypnea musciformis (Wulfen) Lamouroux and Hypnea valentiae (Turner) Montagne in Tanzania". In H. A. Hoppe, T. Levring and Y. Tanaka (eds), Walter de Gruyter Marine Algae. Pharmaceutical Science. New York (1979) 747-782.

[65] - M. Friedlander and N. Zeilikovitsch. "Growth rates, phycocolloid yield and quality of the red seaweeds, Gracilaria sp., Pterocladia capillacea, Hypnea musciformis, and Hypnea cornuta, in field studies in Israel". Aquaculture. 40 (1984) 57-66.

[66] - M. S. Murthy, Y. N. Rao Ramakrishna and D. K. Ghose. "Ecological studies on some agarophytes from Veranal coast (India) I. Effects of aerial conditions on the biomass dynamics". Bot. Mar. 32 (1989) 515-520. 
[67] - R. P. F. Schenkman. "Hypnea musciformis (Rhodophyta) : Ecological influence on growth". J. Phycol. 25 (1989) 192-196.

[68] - F. A. S. Berchez, R. T. L. Pereira and N. F. Kamiya. "Culture of Hypnea musciformis (Rhodophyta, Gigartinales) on artificial substrates attached to linear ropes". Hydrobiologia 260/261 (1993) 415-420.

[69] - J. A. Seoane-Camba. "On the possibility of cultiring Gelidium sesquipedale by vegetative propagation". Proc. of the second workshop of COST 48 Subgroup 1 (1989) 59-68.

[70] - D. Abdellaoui. "Etude biologique de l'agarophyte Gelidium sesquipedale (Turner) Thuret, essais d'induction de la fertilité et de la culture in vitro". (2006) Mémoire de DESS, Université Ibn Tofail, 109 p.

[71] - T. Givernaud, A. El Gourii, A. Mouradi-Givernaud, Y. Lemoine and N. Chiadmi. "Seasonal variations of growth and agar composition of Gracilaria multipartita harvested along the Atlantic coasts of Morocco". Hydrobiologia, 398/399 (1999) 167-172.

[72] - K. T. Bird and J. H. Ryther. "Cultivation of Gracilaria verrucosa (Gracilariales, Rhodophyta) strain G-16 of agar". Proc. Int. Seaweed Symp. 13 (1990) 347-351.

[73] - R. Ugarte and B. Santelices. "Experimental tank cultivation of Gracilaria chilensis in Chile". Aquaculture. 101 (1992) 7-16.

[74] - F. J. Molloy and J. J. Bolton. "The effect of season and depth on the growth of Gracilaria gracilis at Lüdertz, Namibia”. Bot. Mar. 39 (1996).407-413. 